\title{
Green synthesis of silver nanoparticles and biopolymer nanocomposites: a comparative study on physico-chemical, antimicrobial and anticancer activity
}

\author{
RAMASUBBA REDDY PALEM ${ }^{1}$, SHIMOGA D GANESH ${ }^{1}$, ZUZANA KRONEKOVA ${ }^{2}$, \\ MONIKA SLÁVIKOVÁ ${ }^{3}$, NABANITA SAHA ${ }^{1, *}$ (D) and PETR SAHA ${ }^{1}$ \\ ${ }^{1}$ Centre of Polymer Systems, University Institute, Tomas Bata University in Zlin, Tř. T. Bati 5678, 76001 Zlin, Czech \\ Republic \\ ${ }^{2}$ Department for Biomaterials Research, Polymer Institute, Slovak Academy of Sciences, Dubravska cesta 9, 84541 \\ Bratislava, Slovakia \\ ${ }^{3}$ Institute of Virology, Biomedical Centre, Slovak Academy of Sciences, Dubravska cesta 9, 84505 Bratislava, Slovakia \\ *Author for correspondence (nabanita@utb.cz)
}

MS received 3 March 2017; accepted 12 August 2017; published online 29 March 2018

\begin{abstract}
The current report was intended towards comparative study of green-synthesized biogenic Rhubarb silver nanoparticles (RS-AgNPs) and chitosan crosslinked silver nanocomposites (CSHD-AgNCs). The physico-chemical characterization was done by UV-visible, FTIR, scanning electron microscopy (SEM), transmission electron microscopy (TEM), EDX, TGA, XRD and zeta potential $(\zeta)$. The analysis and spectroscopic characterization was done by SEM and TEM and their results reveal that the nanoparticles are spherical in shape, with average size ranges from 5 to $50 \mathrm{~nm}$, and was gathered by face centered cubic (FCC) structure throughout the polymer matrix and stable without any protecting or capping reagents over 450 days. The antimicrobial property of RS-AgNPs and CSHD-AgNCs $(\zeta=+29.6$ and $+32.8 \mathrm{mV})$ was evaluated against E. coli and $S$. aureus and showed an effective inhibitory property. The RS-AgNPs and CSHD-AgNCs were assessed for their anticancer activity against HeLa cell line by MTT method, and it reveals a dose-response activity, time and cell line-dependent cytotoxicity. Based on the results obtained, the RS-AgNPs exhibited higher toxicity over CSHD-AgNCs after $24 \mathrm{~h}$ incubation of HeLa cells with different concentrations and is negligible for the aqueous Rhubarb extract. It was concluded that the changes in anticancer activity towards HeLa cells due to biological activity of silver nanoparticles depend on their method of biosynthesis and their physico-chemical nature.
\end{abstract}

Keywords. Biogenic silver nanoparticles; biopolymer nanocomposites; nanoparticles stability; antimicrobial activity; anticancer activity.

\section{Introduction}

Today, plant-mediated ecofriendly protocols offer a green tool for the synthesis of functional nanomaterials. To overcome the drawbacks of the physico-chemical methods, involving high temperature, high pressure and toxic by-products, it is necessary to adopt the safer alternative methods of metal nanoparticles syntheses. The greatest challenge in nanomaterials study is the development of beneficial green chemistry routes to confirm the inspection protocols for the synthesis of nanomaterials of required size and shape [1]. Recent trends in developing green-triggered technologies for the nanomaterial synthesis portray the enormous importance in material science and technology, also considerable status attributed to a wide variety of potential applications in therapeutic and medical interest [2]. In recent years, researchers have developed a lot of awareness over the environmental and health issues of silver nanoparticles developed from synthetic chemicals (reducing, capping and stabilizing agents).
Because of the remarkable properties (antimicrobial, thermal, optical and electrical), silver nanoparticles used in airborne products (sprays and disinfectants), consumer products used in our daily life (toothpastes, deodorants, shampoos, water purification, air filter and kitchen utensils) and textile industry (synthetic dyes used in wet processing) [3]. The vegetable dyes from plant sources such as roots, leaves, bark and organic sources, such as fungi act as least pollutants, less toxic and less hazardous compared to the carcinogenic nature of synthetic dyes and their intermediates [4]. An attractive possibility of environmental friendly metal nanoparticle synthesis is by utilizing various bio-resources, such as enzymes [5], microorganisms [6], plant parts and plant extracts $[7,8]$, which have numerous advantages over conventional chemical and physical methods.

Cancer is a dangerous class of disease involving abnormal tissue growth, it easily spreads to other parts of the body and in most of the cases it causes death. Chemotherapy is one of the most commonly used treatments to control various types 
of cancer diseases, the existing chemotherapeutic approaches are linked to serve side effects alone or in combination with cytotoxic drugs or by radiation therapy. Recently, the National Cancer Institute, USA, has encouraged the investigation of the potential anticancer activity of plant extracts to reduce the undesirable side effects $[9,10]$. The plant material containing bioactive substances are positively affecting the humans and also improve the health condition as well as good environment. The green plants also include antioxidants which are crucial in combating free radicals. The use of laboratory animals was reduced by increasing the in vitro cytotoxicity testing procedures on cultured tissues and cells. Production of nanomaterials by using living organisms of plant-based materials and other biodegradable polymers is an active part of bio-nanotechnology. The biogenic metal nanoparticles can offer unique interactions with biomolecules both on the external surface and inside the body cells, and this may bring revaluation in cancer therapy [11].

In recent days, there is a progressive need to develop environmentally friendly metal nanoparticles that does not use any toxic chemicals in the synthesis. In the case of polymeric silver nanoparticle synthesis, the reducing agents such as $\mathrm{NaBH}_{4}$, ascorbic acid, citrates [12-16] are used for the reduction of $\mathrm{Ag}^{+}$ions into $\mathrm{Ag}$ nanoparticles, which are toxic in nature. But recently, natural plant extracts were used as reducing agents as well as capping agents to inhibit the agglomeration and provides the inherent stability [17-19] to the synthesized nanoparticles. Currently, the noble metallic nanomaterials are of increasing interest in the recent research domain, due to their interesting inherent properties, exhibiting through completely new and improved characteristic features as compared to the voluminous particles of the bulk materials with specific characteristics, particularly, size distribution and morphology [20,21]. The biosynthesized nanoparticles, such as $\mathrm{Ag}, \mathrm{Au}, \mathrm{Pt}$, are widely used in biomedicine, pharmaceuticals [22,23], bio-labelling, food packaging, cosmetics, catalysis [24,25] and sensory applications [26].

Rhubarb (Rheum rhabarbarum) is a perennial herb, commonly known as garden Rhubarb and belongs to Polygonaceae family. It contains soft fibre-reinforced sandwich structure and grown for its luscious rose red and edible leafy stalks. Its use was first documented around 2100 years ago in a western civilization as an ingredient in numerous Greek and Roman medicines [8,27]. The garden Rhubarb stems are edible due to its good nutritional values such as vitamins (B, $\mathrm{C}$ and $\mathrm{K}$ ), minerals (potassium, calcium and manganese), and also other organic nutrients that make it ideal for keeping good health. In addition, Rhubarb also has some health benefits, used in weight loss, cardiovascular diseases, digestion, bone health, antioxidant, blood circulation, cathartic, laxative properties and is also used as a natural dye. Furthermore, it is used in dessert recipes like pizza, jams, crumbles, custard sweets and pies $[4,8,27,28]$. The Rhubarb stems were also used for pharmaceutical applications, especially in the modern pharmacology against allergies, inflammation and tumours [29]. The Rhubarb stalks were kept fresh for around 2-3 weeks by placing them in polyethylene bags and stored in refrigerator at $0{ }^{\circ} \mathrm{C}$ with $95 \%$ relative humidity. The Rhubarb-mediated silver nanoparticles act as antimicrobial and anticancer agents $[8,30]$.

The aim of the present study is to compose a comparative data among physico-chemical properties (UV-visible, Fourier transform infrared spectroscopy (FTIR), scanning electron microscopy (SEM), transmission electron microscope (TEM), energy dispersive X-ray spectroscopy (EDX), thermogravimetric analysis (TGA), X-ray diffractometer (XRD) and zeta potential), antibacterial and anticancer activities of the Rhubarb-synthesized RS-AgNPs and CSHDAgNCs. The bio-based silver nanoparticles were successfully synthesized by the reduction of aqueous $1 \mathrm{mM} \mathrm{AgNO}_{3}$ solution using aqueous Rhubarb stem extract. From the environmental concern, the method described is relatively simple, inexpensive, and ecofriendly in situ synthesis procedure was developed where active ingredients from Rhubarb extract was attached with silver nanoparticles and chitosan to form RSAgNPs and CSHD-Ag nanocomposites. The cytotoxic action of AgNPs on cervix carcinoma (HeLa) cells was evaluated by counting the number of viable cells after incubation with the Rhubarb extract and silver nanoparticles (RS-AgNPs, CSHD-AgNCs) in different concentrations and time period of exposure was reported.

\section{Materials and methods}

\subsection{Materials}

2.1a Plant and chemicals: The fresh stems of Rhubarb (Rheum Rhabarbarum) were collected from nearby commercial shops of Tomas Bata University campus, Zlin, Czech Republic. Chitosan (CS) (Deacetylation $\geq 75.0 \%$ ), hexamethylene 1,6-diisocyanate, silver nitrate $\left(\mathrm{AgNO}_{3}\right)$, sodium hydroxide were purchased from Sigma-Aldrich Chemical Co., USA. Acetic acid, acetone and sodium metabisulfite were obtained from lach-ner, analytical grade chemicals (Czech Republic) and were used without further purification. Nutrient agar was procured from Hi-Media Chemicals. Deionized water was utilized in all the experiments.

2.1b Microorganisms and cell culture: The evaluation of antibacterial activity was carried out using Escherichia coli (E. coli) and Staphylococcus aureus (S. aureus). The microbial cultures were maintained by Bio-Composite Research Team at Center of Polymer Systems, Tomas Bata University, Zlin, Czech Republic. Human cervix carcinoma (HeLa) cells were obtained from the Institute of Virology BMC SAV (Bratislava, SK). Dulbecco's modified Eagle's medium (DMEM), fetal bovine serum (FBS), streptomycin, penicillin, trypsin-EDTA and L-glutamine were purchased from Gibco (Life Technologies, Grand Island, NY, USA). 

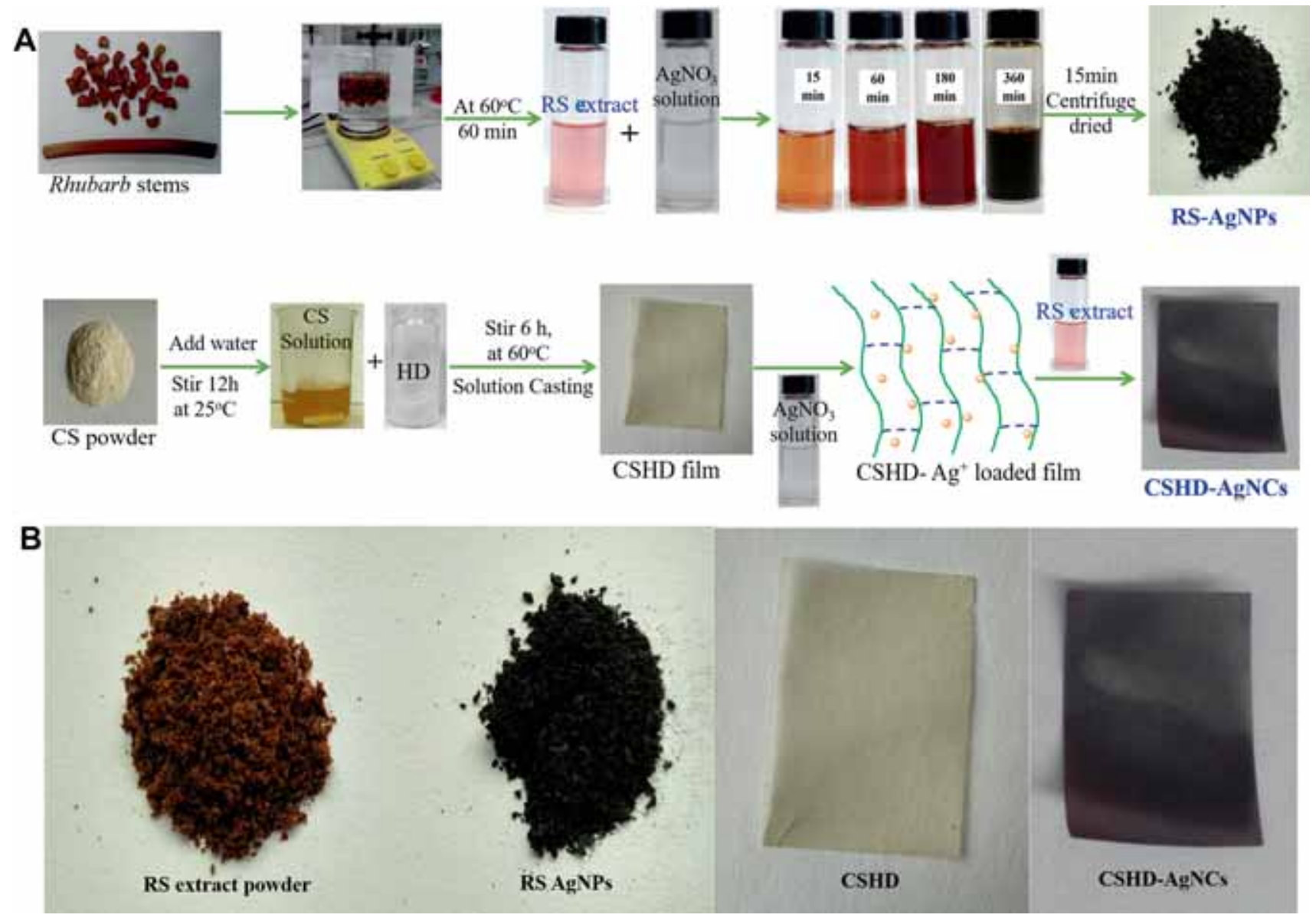

Figure 1. (a) Schematic representation of RS-AgNPs and CSHD-AgNCs synthesis and (b) physical appearance of RS extract, RSAgNPs, CSHD and CSHD-AgNCs.

Dimethyl sulfoxide (DMSO) was purchased from SigmaAldrich (Weinheim, Germany) and 3-(4,5-dimethyldiazol-2yl)-2,5-diphenyltetrazolium bromide (MTT) was purchased from Calbiochem (Merck Millipore, Darmstadt, Germany). All other chemicals are of analytical grade and used without further purification, and deionized water was used throughout the experiments.

\subsection{Synthesis of Rhubarb silver nanoparticles}

The fresh Rhubarb stems (RS) were collected in April month. These stems were cleaned with deionized water and air-dried at room temperature. Twenty grams of nearly $2 \mathrm{~cm}$ dimensions of chopped stems were kept in a beaker containing $100 \mathrm{ml}$ deionized water and boiled for $60 \mathrm{~min}$. The obtained light pink coloured extract was cooled down and filtered with Whatman filter paper no. 1 and extract was stored at $4^{\circ} \mathrm{C}$ for further use. According to previous report [8], briefly, $20 \mathrm{ml}$ of aqueous Rhubarb stem extract was added into $80 \mathrm{ml}$ of aqueous solution of $1 \mathrm{mM} \mathrm{AgNO}_{3}$ solution and this solution was incubated overnight at room temperature in the dark to avoid/minimize photo-activation of $\mathrm{AgNO}_{3}$. The conversion of $\mathrm{Ag}^{+}$to $\mathrm{Ag}^{\circ}$ was confirmed by the colour change of solution from light pink to brown as shown in figure 1a. This was confirmed by using UV-visible spectroscopy and these particles are termed as RS-AgNPs.

\subsection{Synthesis of biopolymer nanocomposites}

We used chitosan as a biopolymer and crosslinked with hexamethylene 1,6-di(amino carboxysulfonate) (HD) and crosslinked chitosan films were obtained by solution casting method. Firstly, chitosan solution $(4 \mathrm{~g}$ per $400 \mathrm{ml})$ in acetic acid (2\%) solution was prepared, and to this, $2.5 \mathrm{wt} \%$ of $\mathrm{HD}$ crosslinker was added as mentioned in previous literature $[30,31]$ and stirred for $6 \mathrm{~h}$ at $60^{\circ} \mathrm{C}$ to achieve a complete uniform solution. The resulting solution was casted on a flat and levelled Petri glass plate. The crosslinked chitosan (CSHD) films were peeled from the Petri glass plate after drying at room temperature for about $36 \mathrm{~h}$ and these films were soaked in deionized water to remove ionic impurities spawned from HD crosslinker. Secondly, the CSHD films (200 mg) were immersed in $30 \mathrm{ml}$ of $1 \mathrm{mM} \mathrm{AgNO}{ }_{3}$ solution at $25^{\circ} \mathrm{C}$ for $12 \mathrm{~h}$. Later, the remaining solution was discarded and freshly 
prepared aqueous Rhubarb stem extract $(20 \mathrm{ml})$ was added into the silver embedded CSHD films. The addition of Rhubarb extract (pink colour) to $\mathrm{AgNO}_{3}$ solution (colourless) led to turn the solution colour into brown red, indicating the formation of AgNPs inside the CSHD films. The formation of CSHD-AgNCs are depicted in figure 1a and designated as CSHD-AgNCs.

The above-mentioned AgNPs synthesis from green Rhubarb plays a pivotal role containing bioactive constituents especially anthraquinones, such as emodin, aloeemodin, undergoes tautomerization, resulting in the reduction of $\mathrm{Ag}^{+}$ ions to Ag particles.

\subsection{Characterization}

Formation of AgNPs and AgNCs was confirmed by ultraviolet-visible spectral analysis. The absorption spectra of RS extract, RS-AgNPs and CSHD-AgNCs were recorded using UV-vis spectrophotometer (VARIAN-EL08043361) in 250-750 nm. FTIR was performed on Thermo Scientific iS5, iD5 ATR spectrometer to detect the possible functional groups in biomolecules that were present in the Rhubarb stem extract and its biopolymer nanocomposites. The morphology and size of nanoparticles were examined using SEM (FEI \& Nova NanoSEM450) operating at an accelerating voltage of 5-15 kV and TEM observations were done by using FEI Tecnai F20 microscope at an accelerating voltage of $200 \mathrm{kV}$. The elemental analysis of nanoparticles was observed by EDX. The TGA of samples (RS-AgNPs, CSHD-AgNCs) was implemented using a TGA thermal analyzer (Q500, TA Instruments, USA). The analysis was performed with temperature range from 25 to $600^{\circ} \mathrm{C}$ in a $50 \mathrm{ml} \mathrm{min}{ }^{-1}$ flow of nitrogen gas at a heating rate of $10^{\circ} \mathrm{C} \mathrm{min}^{-1}$. The crystal nature of the RS-AgNPs and CSHD-AgNCs were investigated using an XRD (PANalytical X'Pert PRO) equipped with PIXcel detector with $\mathrm{CuK} \alpha$ radiation $(\lambda=1.54 \AA$ ) in $2 \theta$ ranging from 25 to $80^{\circ}$. The zeta potential $(\zeta)$ of the RS-AgNPs and CSHDAgNCs was determined using Zetasizer Nano ZS (Malvern) instrument at $25^{\circ} \mathrm{C}$ and scattering angle of $173^{\circ}$.

\subsection{Antimicrobial activity}

The antimicrobial activity of RS-AgNPs and CSHD-AgNCs was effectively accessed against E. coli (CCM 4517) and $S$. aureus (CCM 4516). For this study, a sterile nutrient agar medium $(2.5 \%)$ was autoclaved at a temperature of $121^{\circ} \mathrm{C}$, at a pressure of $15 \mathrm{lb}$ for $25 \mathrm{~min}$ and poured into sterilized Petri plates. After solidification of the media, the agar plates were inoculated with freshly grown $E$. coli and $S$. aureus (1000 $\mu 1$ per each plate) suspensions separately under aseptic condition. Then, a round nutrient solid agar disc $(8 \mathrm{~mm})$ in each plate was replaced with $100 \mu 1$ of the RS-AgNPs solution directly in triplicates. Similarly, by using disc diffusion method, the CSHD-AgNCs discs ( $8 \mathrm{~mm}$ ) were placed on each Petri plate containing nutrient agar media flooded with bacterial suspension. These plates were incubated in static condition at $37^{\circ} \mathrm{C}$ for $24 \mathrm{~h}$ to evaluate the antibacterial activity of RS-AgNPs and CSHD-AgNCs.

\subsection{Anticancer activity}

The anticancer effect of bio-synthesized silver nanoparticles and its biopolymeric nanocomposite films against cervix carcinoma (HeLa) cells was measured by the MTT colorimetric technique. Cells were grown in full growth medium (DMEM, $10 \% \mathrm{FBS}$, penicillin-streptomycin) at $37^{\circ} \mathrm{C}$ in a $\mathrm{CO}_{2}$ incubator with $5 \% \mathrm{CO}_{2}$ and saturated humidity. Briefly, 5000 cells per well were seeded in 96 tissue culture plates, the required quantity of nanoparticle solution was prepared in sterile distilled water by weighing RS-AgNPs and CSHDAgNCs films (the films were rinsed with phosphate buffer solution (PBS) for $1 \mathrm{~min}$ and then placed into the full growth medium $\left(6 \mathrm{~cm}^{2} \mathrm{ml}^{-1}\right)$ according to ISO 10993-12 and incubated for $24 \mathrm{~h}$ at $37^{\circ} \mathrm{C}$ in the sealed tube, then, the full extract was obtained), and diluted to the required concentrations (AgNPs: 10, 50, 100, 150, 200, 250 and $500 \mu \mathrm{g} \mathrm{ml}^{-1}$ and AgNCs: 100, 50, 10 and 1\%) were added and incubated overnight at $37^{\circ} \mathrm{C}$ in the $\mathrm{CO}_{2}$ incubator. After $24 \mathrm{~h}$ incubation, medium was replaced with medium containing MTT at concentration of $0.5 \mathrm{mg} \mathrm{ml}^{-1}$ and incubated for $2 \mathrm{~h}$ at $37^{\circ} \mathrm{C}$ for the reduction of the MTT by normal living cells. Afterwards, MTT medium was removed and DMSO was added. The cytotoxicity effect on HeLa cells was investigated by eluting the MTT with DMSO, and the optical density (OD) values were measured by the plate reader at the wavelength of $595 \mathrm{~nm}$. Similarly, for contact (direct film), cytotoxicity cells at concentration of 100,000 cells per well were seeded in 24 tissue culture plates and incubated overnight. Next day, nanocomposite films with diameter $5 \mathrm{~mm}$ and thickness $<0.5 \mathrm{~mm}$ were placed into wells in quadruplicates $(n=4)$ and incubated for $24 \mathrm{~h}$. After $24 \mathrm{~h}$, films were removed and MTT assay was performed. The experiments were performed in quadruplicates. The percentage of cell viability was calculated using the following equation:

$$
\begin{aligned}
& \text { Cell viability (\%) } \\
& =\frac{\text { Mean OD value of RS-AgNPs (or) CSHD-AgNCs }}{\text { OD value of control cells }} \times 100 \text {. }
\end{aligned}
$$

\section{Results and discussion}

\subsection{Physical appearance}

Figure 1b shows the physical appearance of RS extract powder, RS-AgNPs, CSHD and CSHD-AgNCs. The formation of RS-AgNPs and CSHD-AgNCs (thick brown colour in both) from Rhubarb extract was monitored by absorption spectra. For colloidal stability, the RS-AgNPs and CSHD-AgNCs extract solution was found more stable without protecting reagents over 450 days. After centrifugation of RS-AgNPs, it was stored at room temperature $\left(25^{\circ} \mathrm{C}\right)$. There was no 

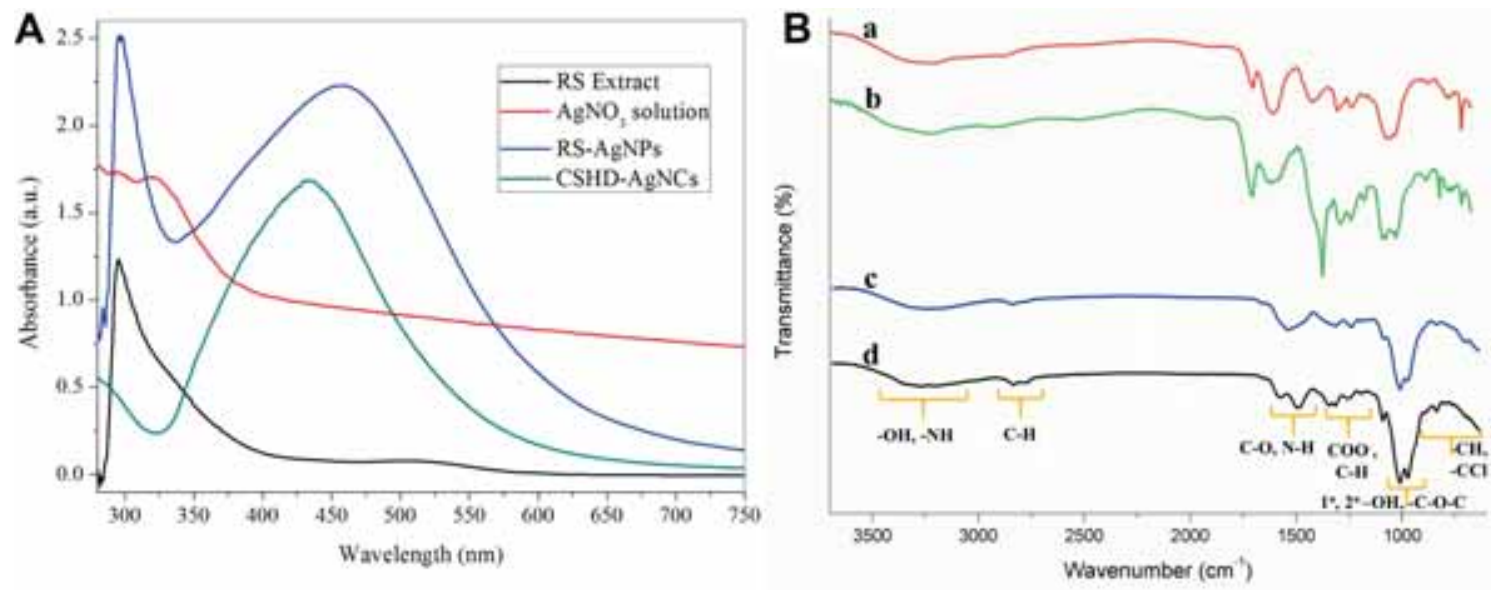

Figure 2. (A) UV-Vis absorption spectra of RS extract, $\mathrm{AgNO}_{3}$, RS-AgNPs and CSHD-AgNCs and (B) FTIR spectra of (a) RS extract (b) RS-AgNPs, (c) CSHD-AgNCs and $(d)$ pure CS.

noticeable change in physical appearance (colour, transparency and flexibility of films) of RS extract powder, RS-AgNPs, CSHD and CSHD-AgNCs after 450 days, this was supported by zeta potential values.

\subsection{UV-Vis spectroscopy}

UV-Vis spectroscopy is a pivotal tool to evaluate the production and stability of metal nanoparticles in an aqueous solution. The RS-AgNPs and CSHD-AgNCs were obtained from an aqueous Rhubarb stem extract as a bio-reductant and appear brown in colour in aqueous medium as well as dry extract powder and in dry film. The absorption spectra of AgNPs and AgNCs formed in single step reaction and media have the absorption maxima in the range of $430-450 \mathrm{~nm}$ due to surface plasmon resonance (SPR) of silver nanoparticles, as shown in figure 2A. The presence of strong SPR peak was well documented for different AgNPs and AgNCs with sizes ranging from 5 to $100 \mathrm{~nm}$ [32]. The plasmon band was shifted to the lower wavelength side (i.e., blue shift) in CSHD silver nanocomposites when compared to RS-AgNPs, which indicates the formation of nanosized particles. The metal nanoparticles with excellent scattering and absorption properties were utilized in biological sensing and detection as well as in the design of colorimetric sensors [33] due to their SPR properties.

\subsection{FTIR spectroscopy analysis}

FTIR analysis was used for the characterization of RS extract, its AgNPs and AgNCs are observed in figure 2B. The RS extract and its AgNPs as shown in figure 2B $(\mathrm{a}, \mathrm{b})$, the absorption peaks at around $3500-3120 \mathrm{~cm}^{-1}$ due to $\mathrm{N}-\mathrm{H}$ stretching from proteins (peptide linkages) of the extract and $\mathrm{H}$-bonded or $\mathrm{O}-\mathrm{H}$ stretching (alcohols, phenols) vibrations, $2900 \mathrm{~cm}^{-1}$ due to $\mathrm{C}-\mathrm{H}$ stretching vibrations, $2517 \mathrm{~cm}^{-1}$ due to $\mathrm{N}-\mathrm{H}$ stretching, $1710-1610 \mathrm{~cm}^{-1}$ due to $\mathrm{C}=\mathrm{O}$ stretching,
$1300 \mathrm{~cm}^{-1}$ due to $\mathrm{C}-\mathrm{C}$ stretching, $1258 \mathrm{~cm}^{-1}$ due to $\mathrm{C}-\mathrm{N}$ stretching (aromatic amine), $1068-1030 \mathrm{~cm}^{-1}$ due to $\mathrm{C}-\mathrm{O}$ stretching $\left(1^{\circ}, 2^{\circ}\right.$ alcohols $), 776 \mathrm{~cm}^{-1}$ due to $\mathrm{C}-\mathrm{H}$ bending, $714 \mathrm{~cm}^{-1}$ due to alkyl halide $(\mathrm{C}-\mathrm{Cl})$ stretching were observed. However, the silver nitrate shows peaks at $733,803,835$ and $1348 \mathrm{~cm}^{-1}$, but after the formation of AgNPs, these peaks were not found. This confirms the formation of AgNPs.

Figure 2B (c, d), shows the characteristic peaks of saccharide structure of the chitosan film and its AgNCs obtained from RS extract. The characteristic peaks at around $3636-$ $3106 \mathrm{~cm}^{-1}$ due to N-H, O-H stretching vibrations, 2913 and $2847 \mathrm{~cm}^{-1}$ due to $\mathrm{C}-\mathrm{H}$ stretching and $\mathrm{N}-\mathrm{H}$ bending vibrations, 1655 and $1564 \mathrm{~cm}^{-1}$ due to amide bond $(\mathrm{C}-\mathrm{O}$ stretching along $\mathrm{N}-\mathrm{H}$ deformation), $1410 \mathrm{~cm}^{-1}$ for $\mathrm{C}-\mathrm{H}$ symmetrical deformation, $1377 \mathrm{~cm}^{-1}$ for $\mathrm{COO}^{-}$stretching and $1076 \mathrm{~cm}^{-1}$ due to $\mathrm{C}-\mathrm{O}-\mathrm{C}$ stretching vibrations in $\beta$-glycosidic unit [30]. The CSHD-AgNCs shows all the corresponding peaks of chitosan. In addition, 1747 and $1150 \mathrm{~cm}^{-1}$ peaks are due to $\mathrm{C}=\mathrm{O}$ stretching (urea) and $\mathrm{C}-\mathrm{N}$ stretching increases and loss of peaks at $1158,1076,1038 \mathrm{~cm}^{-1}$ was observed. The spectrum of the AgNCs, which is shifted to lower wavenumber, confirms the presence of $\mathrm{Ag}$ in CSHD polymer matrix.

From the above results, the probable biomolecules present in the RS extract are responsible for the bio-reduction of $\mathrm{Ag}^{+}$ ions to AgNPs and stabilization of the synthesized AgNPs and $\mathrm{AgNCs}$ for longer period.

\subsection{Electron microscopy analysis}

3.4a SEM and EDX analyses: SEM images of RS extract, RS-AgNPs, CSHD film and CSHD-AgNCs films are shown in figure 3A-D. As shown in figure $3 \mathrm{~A}$, the surface morphology of the RS extract is a sheet-like structure with wrinkles or tiny sprinkled dots, while figure 3B shows RS synthesized AgNPs, which are predominantly spherical in shape, with a large distribution of sizes. Figure 3C shows a smooth 

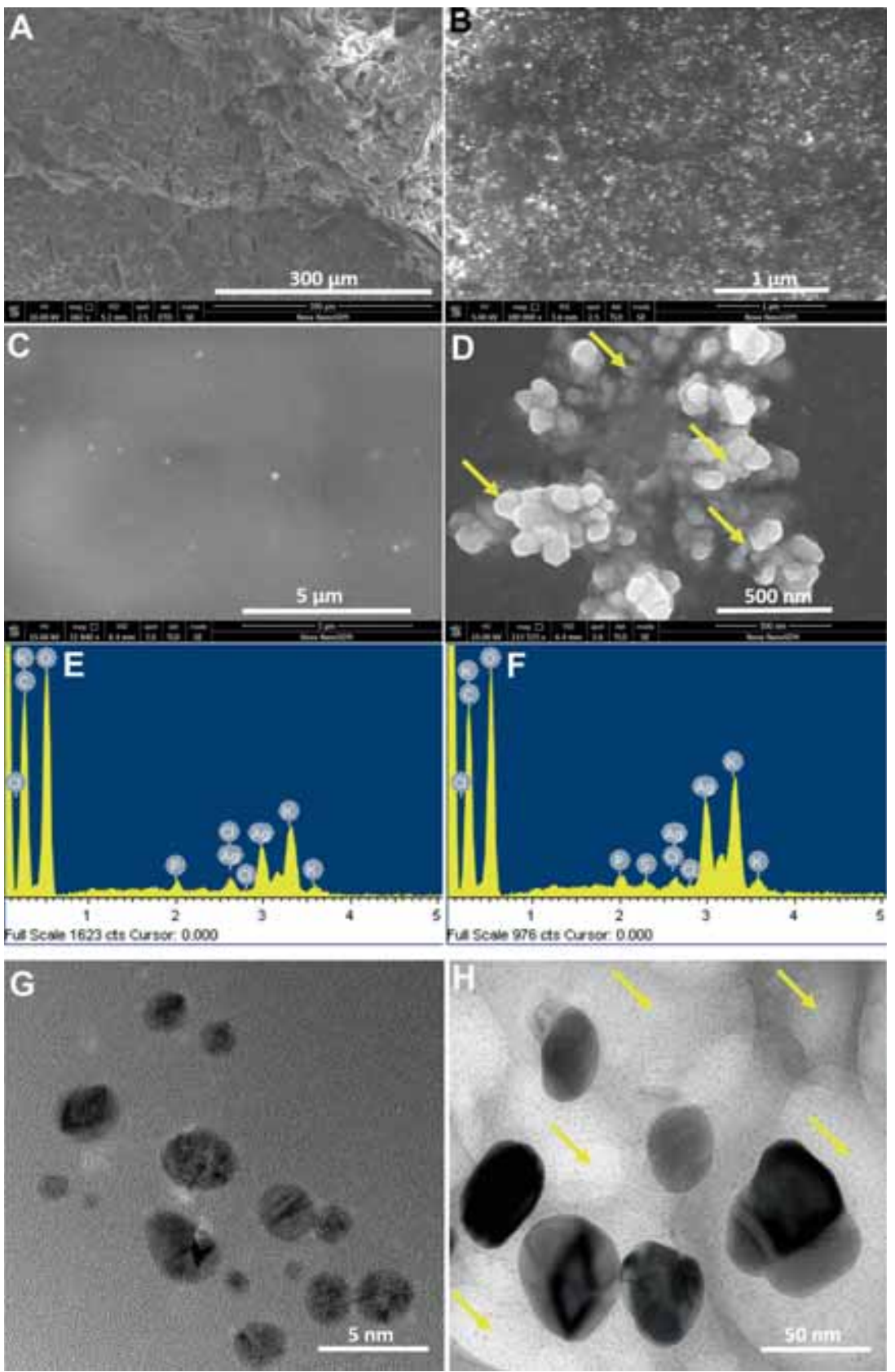

Figure 3. SEM images of (A) RS extract, (B) RS-AgNPs, (C) pure CS, (D) CSHDAgNCs, (E, F) EDX spectra of RS-AgNPs, CSHD-AgNCs and $(\mathbf{G}, \mathbf{H})$ TEM images of RS-AgNPs and CSHD-AgNCs.

surface morphology of pure CS films. But, CSHD-AgNCs synthesized by RS extract showed spherical shape of AgNPs with an average size of 5-50 nm. The AgNPs observed inside the CSHD network are indicated by yellow arrow mark in figure 3D. However, from the SEM results, the morphology of RS-AgNPs and CSHD-AgNCs are mostly spherical, in agreement with the SPR band in UV-Vis spectrum. The average particle size of CSHD-AgNCs is less when compared to the size of RS-AgNPs. In majority, it is reported that the green silver nanoparticles are spherical in shape [7,8], this finding is in agreement with SEM studies.

The EDX spectrum recorded from RS-synthesized AgNPs and CSHD-AgNCs is reported in figure $3 \mathrm{E}$ and F. The EDX spectra revealed a distinct signal peak at $3 \mathrm{keV}$ (optical absorption band due to SPR), this confirms the presence of metallic silver and other elemental signals were recorded possibly due to elements from proteins or acids or alcohol, such as quinones, emodin, rhein and enzymes present within 

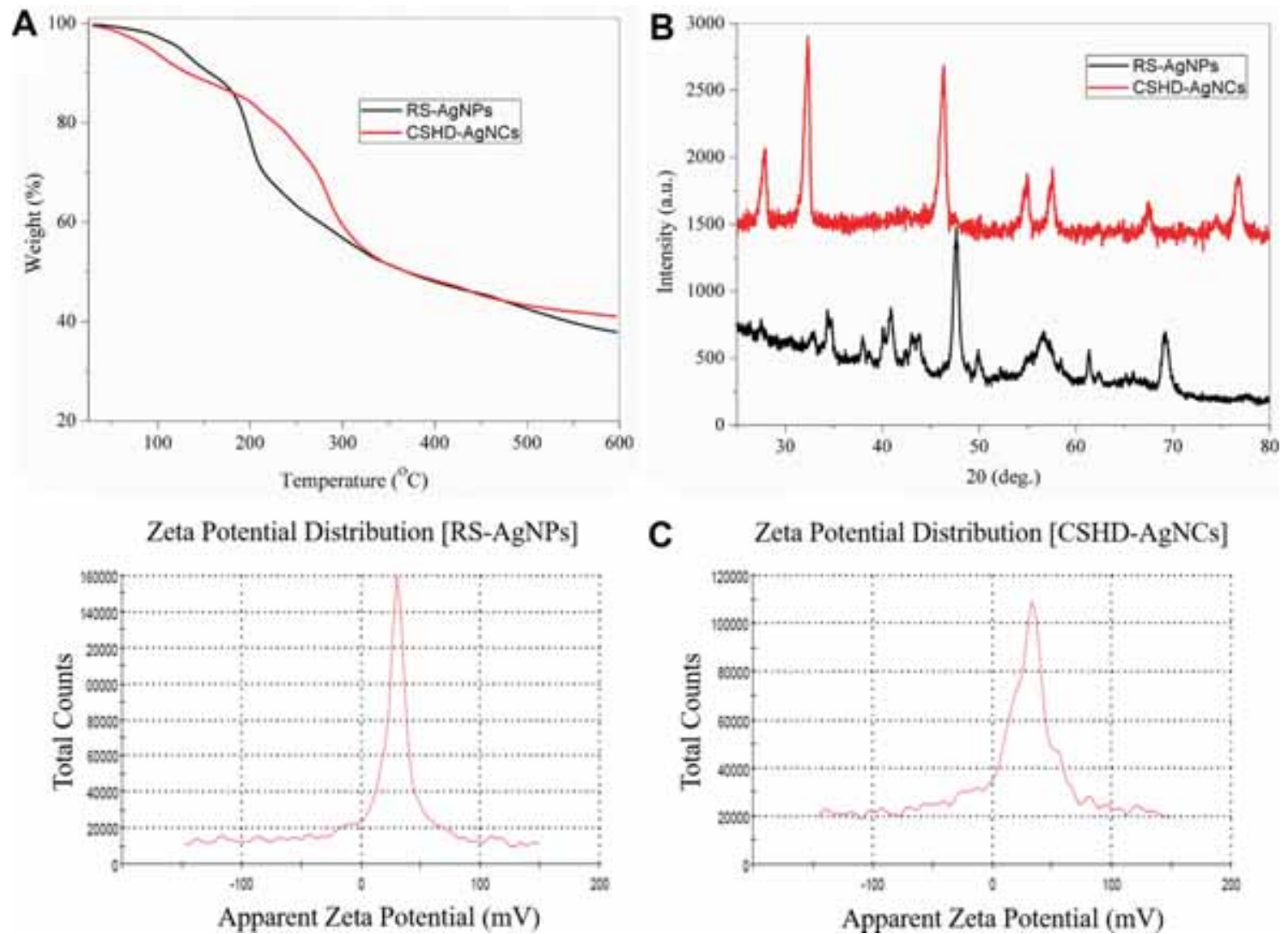

Figure 4. (A) TGA analysis, (B) XRD pattern of RS-AgNPs and CSHD-AgNCs synthesized using Rhubarb extract, and $(\mathbf{C})$ zeta potential of RS-AgNPs and CSHD-AgNCs at room temperature $\left(25^{\circ} \mathrm{C}\right)$ after 450 days of synthesis.

the RS extract. Generally, the metallic AgNPs show typical absorption peak at $\sim 3 \mathrm{keV}[8,30]$.

3.4b TEM analysis: Figure $3 \mathrm{G}$ and $\mathrm{H}$ shows the size, surface morphology and shape of AgNPs from (bioreductant) RS extract, which were characterized and revealed in the TEM micrographs. As shown in figure $3 \mathrm{G}$, the uniform distribution of RS-AgNPs was observed and the average particle size is $<5 \mathrm{~nm}$. In the case of CSHD-AgNCs, the micrographs show spherical AgNPs with size ranging between 5 and $100 \mathrm{~nm}$. In comparison, the TEM image of CSHD-AgNCs with RSAgNPs was clearly indicated uniform and highly dispersed nanoparticles without any aggregation and was deposited in the CSHD matrix (the ultra-small one indicated by yellow arrow mark) as shown in figure $3 \mathrm{H}$.

\section{$3.5 T G A$}

TGA of RS-AgNPs and CSHD-AgNCs results are demonstrated in figure 4A. The thermograms show four and three successive weight losses, respectively. The initial weight loss around $110-160^{\circ} \mathrm{C}$ was about $10 \%$, which were assigned to the evaporation of absorbed water molecules (moisture vapourization) or impurities. The second, third and fourth steps are the consequences of the thermal degradation of the RS-AgNPs starts at around 200, 281 and $458^{\circ}$, respectively.
The total weight loss of RS-AgNPs was about $64 \%$ at $600^{\circ} \mathrm{C}$. Similarly, the second weight loss at $270-384^{\circ} \mathrm{C}$ is due to the degradation of CSHD-AgNCs, and the third weight loss at $435^{\circ}$ and it was continued up to $600^{\circ}$. The total weight loss of CSHD-AgNCs was about $41 \%$ at $600^{\circ} \mathrm{C}$. Therefore, it can be concluded that CSHD-AgNCs exhibited better thermal stability than RS-AgNPs.

\subsection{XRD analysis}

The crystalline nature of Rhubarb-synthesized AgNPs and AgNCs was investigated by XRD analysis as shown in figure 4B. The XRD pattern highlighted that AgNPs and AgNCs formed by the reduction of $\mathrm{AgNO}_{3}$ with RS extract were crystalline in nature. The RS-AgNPs shows diffraction peaks at $32.3,46,54.8,67.54$ and $76.79^{\circ}$ corresponding to (111), (200), (220), (142) and (311) reflections, respectively. Similarly, the CSHD-AgNCs show diffraction peaks at 27.8, $34.4,47.6,56.6$ and $69.2^{\circ}$ corresponding to (111), (200), (220), (311) and (400) planes, respectively. Both AgNPs and AgNCs indicate face centered cubic crystalline structure of metallic silver. The obtained results indicate that silver nanoparticles were finely distributed in the chitosan polymer network and consistent with the results achieved from TEM observation. 


\subsection{Zeta potentials}

Zeta potentials of the RS-AgNPs and CSHD-AgNCs were measured at $25^{\circ} \mathrm{C}$. Zeta potential is the surface charge analysis of the AgNPs to manipulate the stability through electrostatic repulsion between the AgNPs. Using dynamic light scattering (DLS), the measurements were carried out first at 3-4 h after nanoparticles synthesis. The zeta potential of RS-AgNPs $(+29.6 \mathrm{mV})$ is less than the zeta potential of CSHD-AgNCs $(+32.8 \mathrm{mV})$, and are depicted in figure $4 \mathrm{C}$. This indicates that the CSHD-AgNCs are more stable compared to RS-AgNPs. The zeta potential of silver nanoparticles (RS-AgNPs and CSHD-AgNCs) were measured again after 90, 120 and 450 days, shows negligible variations along with single peak in the DLS measurement, which indicates that there was no particle aggregation and were quite stable. However, these silver nanoparticles bound with a thin layer of biomolecules coating on their surface which acts as stabilizing and capping agent. The surface-modified nanoparticles showed improved biocompatibility [30] and intracellular uptake for drug delivery [34]. Therefore, silver nanoparticles were polydispersed without direct contact and stable for long periods. The surface of the silver nanoparticles is strongly dependent on zeta potential due to the absorption property of biomolecules and exopolysaccharides [33].

\subsection{Antimicrobial activity}

The antimicrobial chitosan films were prepared by various techniques and used as a food packaging material, antimicrobial agents in the emerging science as well as in the potential nanomedicine. The antimicrobial experiment details are given in the 'experimental' section and the potential of Rhubarb and chitosan silver nanoparticles were investigated by the agar well diffusion assay, which showed well defined zones against Gram positive strains of $S$. aureus and Gram negative strains of $E$. coli. In this study, we compare the antimicrobial activity of the RS-AgNPs, direct CSHDAgNCs film and CSHD-AgNCs film extract as shown in figure 5. The RS-AgNPs show potential antimicrobial ability against $E$. coli and $S$. aureus. The inhibition effect was enhanced with increasing concentration of RS-AgNPs (i.e., (a) $5 \mu \mathrm{l}$, (b) $50 \mu \mathrm{l}$ and (c) $100 \mu \mathrm{l}$ ), but pure RS extract (control) did not involve in the growth inhibition processes as shown in figure 5A. The antimicrobial activity of the direct CSHD-AgNCs film was measured by agar diffusion method. After $24 \mathrm{~h}$ incubation at $37^{\circ} \mathrm{C}$, the CSHD-AgNCs film ( $8 \mathrm{~mm}$ diameter) did not show any zone of inhibition against $E$. coli and $S$. aureus as shown in figure 5C, when compared to control Petri plates, which contain maximum bacterial growth without silver nanoparticles (figure 5B), this was also supported by previous results $[30,35,36]$. Hence, the extract of Rhubarb-synthesized CSHD-AgNCs film showed zone of inhibition around the disc, which was measured and recorded. The antimicrobial effect of CSHD-AgNCs films are depicted in figure 5D. According to the literature, chitosan itself with some antimicrobial property, this property is still not fully elucidated, but different mechanisms were proposed. Chitosan did not show any swelling property, due to this reason, we have synthesized crosslinked chitosan films. Hence,

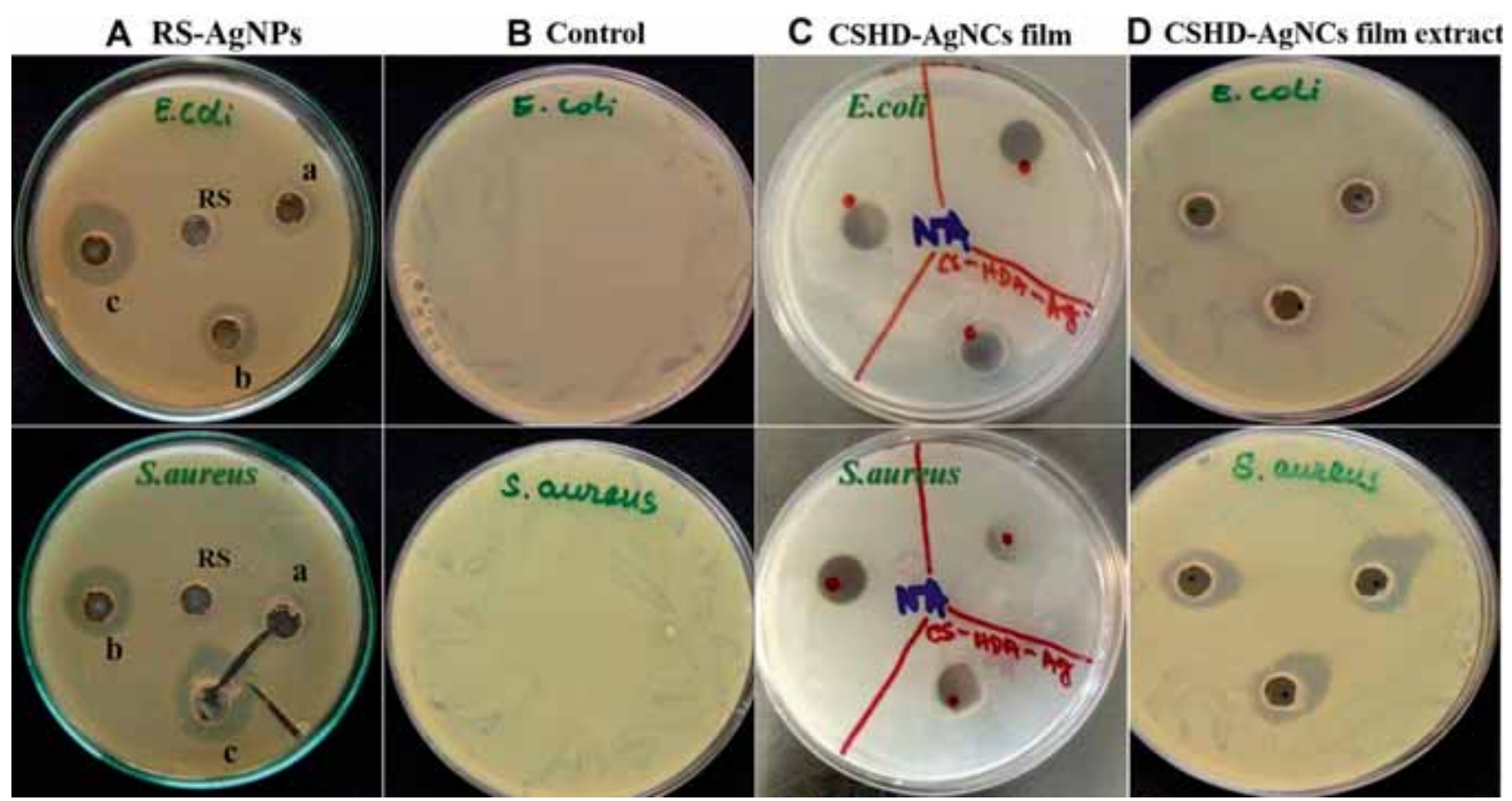

Figure 5. Antimicrobial activity of (A) RS-AgNPs, (B) control, (C) direct CSHD-AgNCs film and (D) CSHD-AgNCs film extract against $E$. coli and $S$. aureus. 

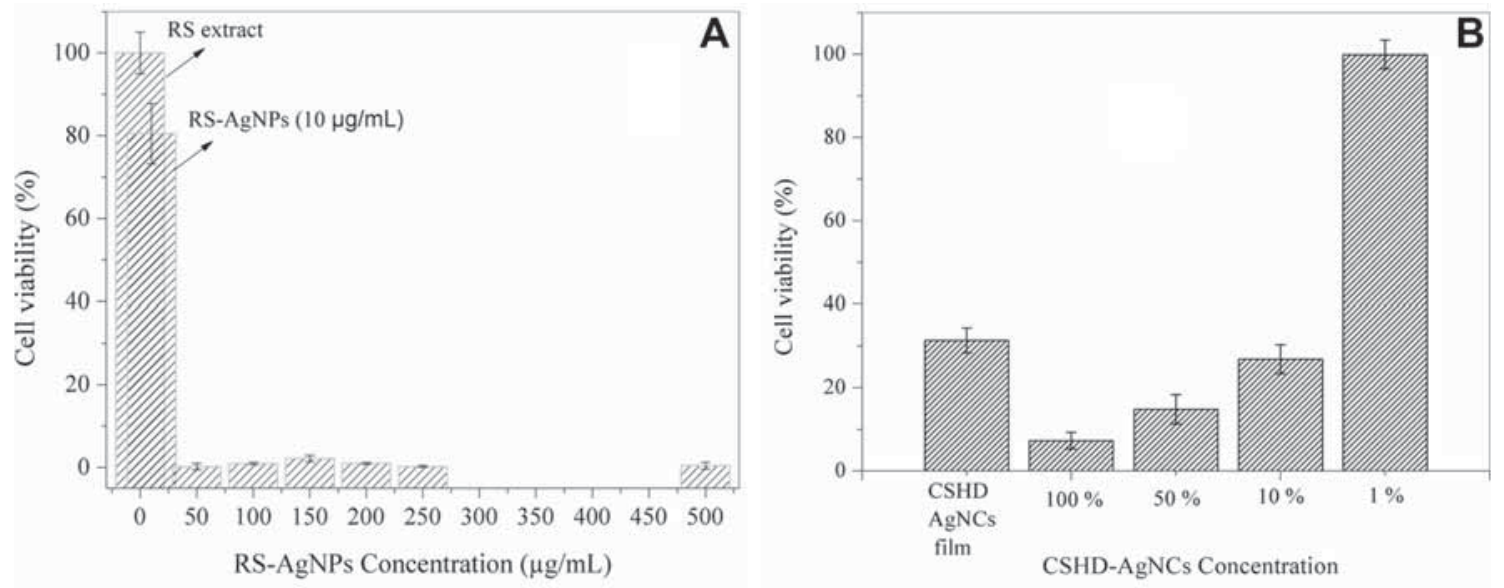

Figure 6. Cytotoxic effect of (A) RS-AgNPs and (B) CSHD-AgNCs on HeLa cells.

the crosslinked CSHD film with three-dimensional network containing swelling property, it is useful for drug delivery, wound dressing and food packaging applications. The direct CSHD-AgNCs film did not reveal any noticeable zone of inhibition against $E$. coli and $S$. aureus, as the AgNPs are bound inside the matrix. In case of CSHD-AgNCs film extract, the release of silver particles are abundant and are in direct contact with the cell wall (it contains peptidoglycan, proteins and lipids) to destabilize it and alter cell permeability resulting in clear zone of inhibition. In recent years, the bio/green, such as Azadirachta indica [37], mint leaf [38], Limonia acidissima [7], Rhubarb stem [8], synthesized polymer-based 3D network silver nanocomposites are considered as non-toxic, environmentally friendly antimicrobial agents, food packaging material, wound dressing material and drug delivery applications [39].

\subsection{Anticancer activity}

Once the preparation of RS extract, RS-AgNPs and CSHDAgNCs was done, it was fully characterized by various physio-analytical techniques and focussed our attention to study their potential use as an antiproliferative agent against cervix carcinoma (HeLa) cancer cell lines. The cell viability studies were compared between RS-AgNPs and CSHDAgNCs. It was noted from the plots (figure $6 \mathrm{~A}$ and $\mathrm{B}$ ) that the effective cell inhibition was observed for the higher concentrations of RS-AgNPs $\left(>10 \mu \mathrm{g} \mathrm{ml}^{-1}\right)$ and CSHD-AgNCs (at higher concentrations, above $>10 \%$ ) evaluated by MTT assay.

Recently, our research team published first report on normal 3T3 fibroblasts and cervix carcinoma (HeLa) cancer cell line of chitosan biopolymer silver nanocomposites and Rhubarbsilver nanoparticles as a promising alternative for traditional anticancer therapeutics [8,30]. Several factors influence the toxic effects of AgNPs, such as concentration, time of exposure and particle size. It was clear from the previous studies that biogenic AgNPs show dependent cytotoxic effects on selected cell lines [40].

Moreover, the influencing factors, such as shape, surface coatings/charge, zeta potential might also be crucial in the AgNPs toxicity. An increase in the absolute zeta potential usually leads to increased nanoparticle uptake in comparison to less-charged nanoparticle of the same size [40]. The interaction of AgNPs with cell membrane is profoundly depends on the size of the nanoparticles and its reactivity towards organic groups of biomolecules. Cellular uptake of nanoparticles provoke the oxidative stress by generating reactive oxygen species, which causes nanoparticles to cross cellular membrane easily and interact with DNA directly or indirectly. Although, the exact mechanism for this phenomenon of interaction is still not clear [41].

The cytotoxic activity of CSHD-AgNCs film extract (100, 50,10 and $1 \%$ ) shows a dose-dependent response relationship and only the highest dilution ( $1 \%$ extract) leads to cell viability compared to untreated control cells [30]. The CSHD-AgNCs film, significantly inhibit the cell growth by more than $70 \%$. In contrast, the presence of 100,50 and $10 \%$ of nanocomposite particles significantly inhibited the cell line growth. From the above results, RS-AgNPs shows higher cell inhibition compared to CSHD-AgNCs. Moreover, RS extract did not show any noticeable anticancer activity. However, further clinical trials are necessary to fully understand the potential anticancer activity of RS-AgNPs CSHD-AgNCs to explore their therapeutic applications in bioscience and nanomedicine.

\section{Conclusion}

In summary, we have fabricated a simple, inexpensive and ecofriendly route to synthesize biogenic Rhubarb silver nanoparticles (RS-AgNPs) as well as crosslinked chitosan silver nanocomposites (CSHD-AgNCs). The formation of silver nanoparticles was confirmed by UV-Vis 
spectroscopy and their functional groups were identified by FTIR analysis. The surface morphology of RS extract, CS, RS-AgNPs and CSHD-AgNCs clearly identified the changes before and after the formation of silver nanoparticles by SEM analysis. The presence of metallic silver was also confirmed by EDX analysis. The colloidal suspension of RS-AgNPs and CSHD-AgNCs are stable for several months without any protecting or capping reagents, this was supported by zeta potential values. The nanoparticles formed are spherical in shape, sizes range from 5 to $50 \mathrm{~nm}$ with crystalline nature. The RS-AgNPs are freely suspended in water and CSHD-AgNCs showed remarkable swelling property, this will be effectively useful in drug delivery and wound-dressing applications. The CSHD-AgNCs films are flexible and thermally more stable than RS-AgNPs. The RSAgNPs have potent antimicrobial activity against $E$. coli and $S$. aureus, when compared to the CSHD-AgNCs films. The stable biogenic RS-AgNPs embedded wound dressings may be helpful to reduce wound bioburden as well as healing time. The in vitro cytotoxicity assay against HeLa cells, the Rhubarb synthesized CSHD-AgNCs and RS-AgNPs triggered certain anticancer signalling pathways, leading to a rapid inhibition of HeLa cell viability compared to RS extract. This study might elaborate the potential use of RS-AgNPs and biopolymer nanocomposites (CSHD-AgNCs) for drug delivery, food packaging and cancer-related therapeutic applications.

\section{Acknowledgements}

We are grateful to the Ministry of Education, Youth and Sports of the Czech Republic-NPU Program I (LO1504) and Slovak Grant Agency VEGA for the financial support in the Project 2/0124/18. We are also thankful to Department of Material Science and Nanotechnology (IISc) Bangalore, India, for TEM analysis.

\section{References}

[1] Nel A E, Mädler L, Velegol D, Xia T, Hoek E M V, Somasundaran P et al 2009 Nat. Mater. 8543

[2] Sharma N C, Sahi S V, Nath S, Parsons J G, Gardea-Torresdey J L and Pal T 2007 Environ. Sci. Technol. 415137

[3] Leo B F, Chen S, Kyo Y, Herpoldt K L, Terrill N J, Dunlop I E et al 2013 Environ. Sci. Technol. 4711232

[4] Osman H 2014 World Appl. Sci. J. 29592

[5] Willner I, Baron R and Willner B 2006 Adv. Mater. 181109

[6] Jebali A, Ramezani F and Kazemi B 2011 J. Cluster Sci. 22 225

[7] Ramasubba Reddy P, Krishna Rao K S V, Madhusudana Rao K, Sivagangi Reddy N and Eswaramma S 2015 J. Drug Deliv. Sci. Technol. 29181

[8] Reddy P R, Ganesh S D, Saha N, Zandraa O and Sáha P 2016 J. Nanotechnol. 20161
[9] Visweswara Rao P, Nallappan D, Madhavi K, Rahman S, Wei L J and Gan S H 2016 Oxid. Med. Cell Longev. 20161

[10] Cai Y, Luo Q, Sun M and Corke H 2004 Life Sci. 74 2157

[11] Raghunandan D, Ravishankar B, Sharanbasava G, Mahesh D B, Harsoor V, Manjunath S et al 2011 Cancer Nanotechnol. 2 57

[12] Sileikaite A, Prosycevas I, Puiso J, Juraitis A and Guobiene A 2006 Mater. Sci. 12287

[13] Durga Praveena V and Vijaya Kumar K 2014 Ind. J. Adv. Chem. Sci. 2171

[14] Malina D, Sobczak-Kupiec A, Wzorek Z and Kowalski Z 2012 Dig. J. Nanomater. Bios. 71527

[15] Kim K A, Cha J R and Gong M S 2013 Bull. Korean Chem. Soc. 34505

[16] Al-Thabaiti S A, Al-Nawaiser F M, Obaid A Y, Al-Youbi A O and Khan Z 2008 Colloids Surf. B 67230

[17] Kharissova O V, Dias H V R, Kharisov B I, Pérez B O and Jiménez Pérez V M 2013 Trends Biotechnol. 31240

[18] Malarkodi C, Rajeshkumar S, Paulkumar K, Vanaja M, Gnanajobitha G and Annadurai G 2014 Bioinorg. Chem. Appl. 20141

[19] Sharma V K, Yngard R A and Lin Y 2009 Adv. Colloid. Interf. Sci. 14583

[20] Ankanna S, Prasad T N V K V, Elumalai E K and Savithramma N 2010 Dig. J. Nanomater. Bios. 5369

[21] Mahdieh M, Zolanvari A, Azimee A S and Mahdieh M 2012 Sci. Iran. 19926

[22] Singh M, Kalaivani R, Manikandan S, Sangeetha N and Kumaraguru A K 2013 Appl. Nanosci. 3145

[23] Galdiero S, Falanga A, Vitiello M, Cantisani M, Marra V and Galdiero M 2011 Molecules 168894

[24] Burda C, Chen X, Narayanan R and El-Sayed M A 2005 Chem. Rev. 1051025

[25] Yallappa S and Manjanna J 2014 J. Cluster Sci. 251449

[26] Gomez-Romero P 2001 Adv. Mater. 13163

[27] A brief history of Rhubarb in western civilization, http:// www.highaltituderhubarb.com/Pages/history.aspx. Accessed 16 December 2016

[28] Health benefits of Rhubarb https://www.organicfacts.net/ health-benefits/fruit/rhubarb.html. Accessed 16 December 2016

[29] Li H, Yang T, Zhou H, Du J, Zhu B and Sun Z 2017 Front. Pharmacol. 7536

[30] Ramasubba Reddy P, Nabanita S, Ganesh S D, Zuzana K, Monika S and Petr S 2017 Int. J. Polym. Mater.,https://doi. org/10.1080/00914037.2017.1291516

[31] Welsh E R, Schauer C L, Qadri S B and Price R R 2002 Biomacromolecules 31370

[32] Chen D, Qiao X, Qui X and Chen J 2009 J. Mater. Sci. 44 1076

[33] Ravindran A, Chandran P and Sudheer Khan S 2013 Colloids Surf. B 105342

[34] Benyettou F, Rezgui R, Ravaux F, Jaber T, Blumer K, Jouiad M et al 2015 J. Mater. Chem. B 37237

[35] Krishna Rao K S V, Ramasubba Reddy P, Lee Y I and Kim C 2012 Carbohydr. Polym. 87920

[36] Tripathi S, Mehrotra G K and Dutta P K 2001 Bull. Mater. Sci. 3429

[37] Jayaramudu T, Raghavendra G M, Varaprasad K, Sadiku R and Raju K M 2013 Carbohydr. Polym. 922193 
[38] Varaprasad K, Reddy G S M, Jayaramudu J, Sadiku R, Ramam K and Ray S S 2014 Biomater. Sci. 2257

[39] Jayaramudu T, Raghavendra G M, Varaprasad K, Mohana Raju K, Sadiku E R and Kim J 2016 J. Appl. Polym. Sci. 133 43921
[40] Mónica G, Ravishankar B, Elena A, Félix S, Dolores M M, Javier H F et al 2016 Materials 9325

[41] Jeyaraj M, Sathishkumar G, Sivanandhan G, Mubarak Ali D, Rajesh M, Arun R et al 2013 Colloids Surf. B: Biointerf. 106 86 